\title{
Experimental Evaluation of Parameterized Nonlinear MPC Applied to PEM Fuel Cell
}

\author{
Cédric Damour ${ }^{\circledR}$, Dominique Grondin, Michel Benne, Brigitte Grondin-Perez, Jean-Pierre Chabriat
}

LE$^{2}$ P-EnergyLab, EA 4079, University of La Reunion, Saint Denis, France

Email: cedric.damour@univ-reunion.fr

How to cite this paper: Damour, C., Grondin, D., Benne, M., Grondin-Perez, B. and Chabriat, J.-P. (2020) Experimental Evaluation of Parameterized Nonlinear MPC Applied to PEM Fuel Cell. Engineering, 12, 99-116. https://doi.org/10.4236/eng.2020.122010

Received: November 7, 2019

Accepted: February 24, 2020

Published: February 27, 2020

Copyright $\odot 2020$ by author(s) and Scientific Research Publishing Inc. This work is licensed under the Creative Commons Attribution International License (CC BY 4.0).

http://creativecommons.org/licenses/by/4.0/

(c) (i) Open Access

\begin{abstract}
This paper proposes a parameterized nonlinear model-based predictive control (NMPC) strategy to tackle the oxygen excess ratio regulation challenge of a proton exchange membrane fuel cell. In practice, the most challenging part regarding NMPC strategies remains the on-line implementation. In fact, NMPC strategies, at least in their basic form, involve heavy computation to solve the optimization problem. In this work, a specific parameterization of control actions has been designed to address this limitation and achieve on-line implementation. To assess the effectiveness and relevance of the proposed strategy, the controller has been implemented on-line, experimentally validated on a real fuel cell and compared to the built-in controller. Performance of the parameterized NMPC controller in terms of setpoint tracking accuracy, disturbances rejection and computational cost, have tested under several control scenarios. Experimental results have shown the excellent tracking capability, disturbances rejection ability and low computational cost of the NMPC controller, regardless of the operating conditions. Moreover, compared to the built-in controller the proposed strategy has demonstrated better disturbances rejection capability. Overall, the proposed parameterized NMPC controller appears as an excellent candidate to address the oxygen excess ratio regulation issue.
\end{abstract}

\section{Keywords}

Control Application, Parameterized Nonlinear Model Predictive Control, Proton Exchange Membrane Fuel Cell

\section{Introduction}

For the last decade, to reduce greenhouse gas emissions and fossil fuel dependence, numerous renewable energy technologies have been studied. In regard to 
renewable energies, one of the primary drawbacks is the variability of the supply flows, which raises the key issue of energy storage to counteract the intermittent nature of their conversion. In this context, the hydrogen vector represents a promising alternative, as long as the hydrogen is produced from a renewable energy technology (e.g. electrolytic hydrogen obtained using photovoltaic energy), and stored to optimize potential gaps and surplus of intermittent production. Downstream the hydrogen chain, electricity is generated from fuel cells, genuine zero-emission power generators. Due to their high power density and low operating temperature, proton exchange membrane fuel cells (PEMFC) have proved to be the most suitable fuel cell technology for both transportation and stationary applications [1] [2] [3].

However, several bolts still remain to be removed to improve their reliability and energy conversion, reduce their cost or extend their lifetime. Among them, one of the most important is related to their control. Indeed, global efficiency improvement, optimal hydrogen and air consumption, and reliable and accurate power response remain challenging control goals.

Numerous control strategies have been reported in the literature for PEMFC systems, ranging from PID controllers [4] [5] [6], state feedback linearizing or differential flatness approaches [7] [8], dynamic neural network controllers [9] [10], linear quadratic Gaussian (LG) controllers [11], to model predictive control strategies [12]. Due to its ability to take into account dynamic nonlinearities of the process, to handle just as well state and input constraints as economical constraints, nonlinear model-based predictive control (NMPC) strategy appears as a promising candidate regarding PEMFC control.

Several works dealing with benefits of predictive control strategies regarding PEMFC control have been reported. Wu et al. [13] proposed a multi-loop nonlinear predictive control strategy using a reduced order model to regulate oxygen excess ratio and stack temperature of a fuel cell. However, the controller has only been tested in simulation environment, and as emphasized by authors some devices and design, such as hydrogen storage or power management, have not been considered. Shokuhi-Rad et al. [14] designed an approximate predictive control strategy to regulate the output voltage of a PEMFC. This approach, based on an instantaneous linearization of a neural network model, has been tested in simulation environment, and appeared to be an interesting alternative to achieve real-time control. Gruber et al. [15] developed a model based predictive strategy to regulate the oxygen stoichiometric ratio using compressor motor voltage as manipulated variable. Experimental results showed that the designed controller stabilized the oxygen stoichiometric ratio around the target value five times faster than the built-in controller. Ziogou et al. [16] proposed a nonlinear model-based predictive control (NMPC) approach to track variable load demands while minimizing hydrogen consumption and avoiding oxygen starvation. To achieve on-line application, these authors developed a tailor-made optimization strategy that discretizes control variables and state variables. The proposed approach has 
been experimentally confirmed and exhibited good performance in terms of tracking capability and computational time efficiency.

Regarding nonlinear model-based predictive control strategies, the modeling step remains essential and mandatory for the control design. In the literature, a wide variety of models, either mechanistic or black-box, with different levels of complexity, can be consulted. Regarding mechanistic approaches, numerous one-dimensional and multi-dimensional models have been proposed [17]-[28]. However, due to their complexity and high computational time, most of these models are not suitable for real-time control purposes. In this context, black-box approaches appear as a promising option to model the PEMFC system. Several works dealing with the benefits of artificial neural network (ANN) regarding PEMFC modeling have been reported. Saengrung et al. [29] investigated the relevance of ANNs as predictive models of a commercial PEMFC system (1.2 kW). The authors developed and compared the performance of back-propagation (BP) and radial basis function (RBF) algorithms in terms of speed and accuracy to predict the stack voltage and current, using the air flow rate and the stack temperature measurements as inputs. Sisworahardjo et al. [30] developed an ANN model of a portable PEMFC $(100 \mathrm{~W})$ considering stack temperature and current as inputs, and the hydrogen flow rate, the stack voltage and power as outputs. The authors reported that, except the transient voltage response of the ANN model, which is faster than the actual fuel cell, the performance of the ANN model in terms of speed and accuracy are quite satisfactory. In Chavez-Ramirez et al. [31], the authors proposed an ANN model of a high power PEMFC (5 kW) using seven inputs to predict the cell voltage and the cathode temperature. The authors reported a maximum prediction error of $9.4 \%$ in the stack voltage and $5.6 \%$ in the stack temperature measurements.

To ensure optimal performance of PEMFC, one parameter, namely oxygen excess ratio, requires special attention. The oxygen excess ratio or stoichiometric ratio represents the ratio of inlet oxygen flow to reacted oxygen flow and is widely used to guarantee safety and to reach a high performance. Arce et al. [32] showed that the oxygen excess ratio has a fundamental influence on the efficiency and the safety of the fuel cell system. A Poor control of this variable can increase the starvation phenomenon probability. The oxygen starvation phenomenon occurs when the oxygen partial pressure falls below a critical level at any location at the cathode [33]. It has been experimentally demonstrated that the oxygen starvation phenomenon can cause damages to the electro-catalyst of the fuel cell, as well as reducing its performance [34]. In this context, preventing oxygen starvation to ensure optimal conversion efficiency and avoid performance deterioration remains a challenging control goal.

In this paper, a real-time implementable nonlinear model-based predictive control (NMPC) strategy is developed to tackle the oxygen excess ratio regulation issue. In practice, the most challenging part regarding NMPC strategies remains the on-line implementation. In fact, NMPC strategies, at least in their basic form, 
involve heavy computation to solve the optimization problem. In this work, a specific parameterization of control actions is designed to address this limitation and achieve on-line implementation. This parameterization allows to significantly decrease the optimization problem dimension and so the computational time required to solve the optimization problem. Regarding real-time control goal, and according to its short computational time compared to classical mechanistic model and its low sensitivity to noise, an artificial neural network (ANN) model is used as internal model to predict the PEMFC behavior.

The rest of this paper is organized as follows: The experimental setup of the fuel cell unit is presented in Section 2. Section 3 is dedicated to the control design - the ANN model predictor is designed and experimentally validated-and the real-time implementable NMPC scheme dedicated to PEMFC oxygen excess ratio control is detailed. Experimental validation of the proposed control strategy is presented in Section 4. Eventually, conclusions and prospects are drawn in Section 5 .

\section{Experimental Setup}

In the present study, a $50 \mathrm{~cm}^{2}$ single home fuel cell assembling, with a commercial membrane electrode assembly (MEA) from Paxitech, is used. The fuel cell is assembled with Teflon glass fiber as seal joints and clamped at $11 \mathrm{Nm}$ bolt torque. MEA used for this work is composed of symmetric electrodes $\left(0.5 \mathrm{mgPt} \cdot \mathrm{cm}^{-2}, \mathrm{Pt}\right.$ $40 \mathrm{wt} \% / \mathrm{C})$ with a Nafion ${ }^{\circ}$ N115 membrane. Air and pure hydrogen are used as oxidant and fuel, respectively. All experiments are performed on a Fuel Cell Test Station (FCTS) manufactured by Fuel Cell Technologies, Inc.

The FCTS provides control of temperature, flow rate, pressure and humidity for both feed gases. The bench integrates an AC-Impedance measurement system and a programmable DC electronic load. Inlet gas flow rates are controlled using two mass flow controllers, located before the humidifier. Humidification and fuel cell temperatures are controlled using temperature controllers. Reactant gases are humidified through external water tanks, and desired gas humidification value is reached through the regulation of the water temperature in the humidifiers. Output pressures are regulated using backpressure controllers designed to operate between 0 and $400 \mathrm{kPa}$. An electronic load measures cell output voltage and current (Figure 1).

The Supervisory Control and Data Acquisition (SCDA) system relies on a proprietary graphical user interface composed of a set of modular virtual instruments (VI) developed with LabView ${ }^{\mathrm{TM}}$ graphical software. This makes the experimental unit a flexible environment, allowing to perform a wide range of tests based on various configuration settings. Since VI's can easily be modified and/or replaced, alternative monitoring and control strategies can be integrated to the SCDA system. As regards to innovative control strategies, a set of VIs makes it possible to replace the original built-in controller by model-based approaches. 


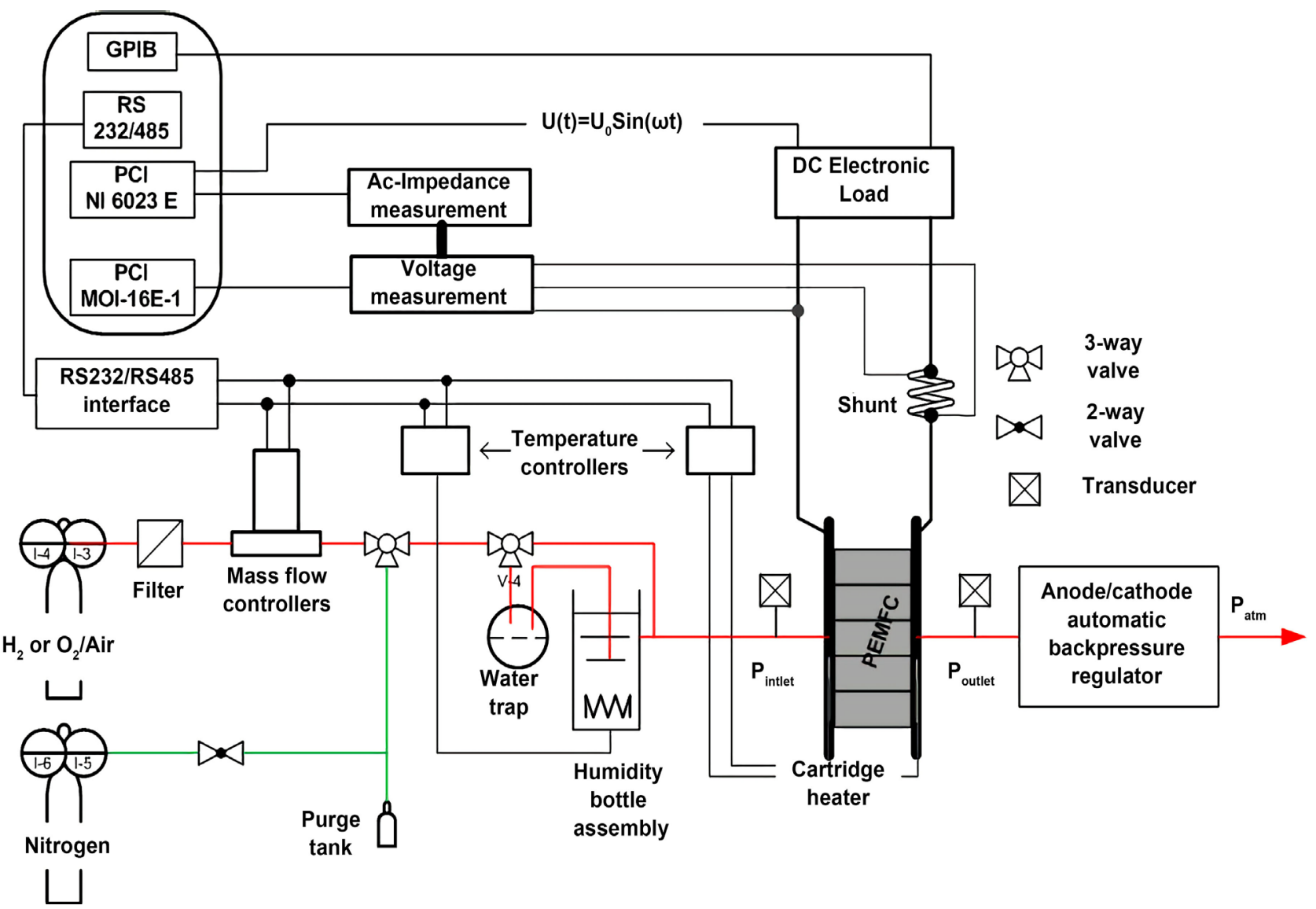

Figure 1. Simplified process and instrumentation diagram of the FCTS system.

\section{Controller Design}

Regarding NMPC control strategies, the first step is to design a model of the system. This model is expected to predict the system behavior several steps ahead. Among the various PEMFC models that can be consulted in the literature, a very few are dedicated to control purposes. In real-time control context, most of complex and heavy computations mechanistic models cannot be considered. Recently, several works emphasize the interest of ANN to model PEMFC systems. Here, due to its short computational time, its low sensitivity to noise and its reliability an ANN model is designed and used as predictive model.

\subsection{ANN Control Oriented Model}

The proposed control strategy involves an ANN model of the PEMFC used to predict the system output several steps ahead.

The oxygen excess ratio, namely $\lambda_{\mathrm{O}_{2}}$, denotes the ratio of oxygen supply to oxygen consumed in the cathode. $\lambda_{\mathrm{O}_{2}}$ is a function of the inlet air flow rate at the cathode $\dot{m}_{a i r}$, the load current $I$, the pressure $P_{c a, i n}$, the temperature $T_{c a, i n}$, the relative humidity $\phi_{c a, i n}$, the number $n$ of cells of the fuel cell stack, the Faraday constant $F$, the oxygen mole fraction $y_{O_{2}, c a, i n}$ and the molar masses $M_{\mathrm{O}_{2}}, M_{N_{2}}$ and $M_{v}$ of oxygen, nitrogen and vapor, respectively. 


$$
\lambda_{\mathrm{O}_{2}}=\frac{\dot{m}_{a i r}}{I} f\left(P_{c a, i n}, T_{c a, i n}, \phi_{c a, i n}, n, F, y_{O_{2}, c a, i n}, M_{O_{2}}, M_{N_{2}}, M_{v}\right)
$$

However, del Real et al. [35] and Gruber et al. [15] showed that $\lambda_{\mathrm{O}_{2}}$ mainly depends on the inlet air flow rate at the cathode $\dot{m}_{\text {air }}$ and the load current $I$. Therefore, regarding control purposes, a simplified model of $\lambda_{\mathrm{O}_{2}}$ based only on these two main variables can be written as:

$$
\lambda_{\mathrm{O}_{2}}=f\left(\dot{m}_{\text {air }}, I\right)
$$

In this paper, an ANN model is designed to predict $\lambda_{\mathrm{O}_{2}}$ several steps ahead, once the load current $I$ and the inlet air flow rate $\dot{m}_{\text {air }}$ are available. In practice, the load current is a measured disturbance, whereas the inlet air flow rate is the manipulated variable.

Regarding real-time implementation goal, the computational speed of the model is a key component. The model is expected to predict the system output with sufficient accuracy, while avoiding heavy computations. To address this problem, several ANN architectures have been investigated to determine which one provides the best trade-off between prediction accuracy and computational speed. Finally, a fully connected three layers network is designed. The hidden layer has five neurons with a tangent sigmoid activation function $\varphi_{1}$, whereas the output layer has one neuron using a linear activation function $\varphi_{2}$.

Identification (training phase) and validation of the ANN model are performed on two different sets of data collected on the PEMFC system presented in Section 2 and sampled with a 3 seconds period. Regarding the poor extrapolation performance of ANN models, the data set used for the training phase has to be chosen wisely. In this aim, the training data set, used to identify the ANN model, covers the whole operating conditions and ranges from minimum to maximum inputs/outputs values. Experiments are performed for different values of load current (ranging from $5 \mathrm{~A}$ to $20 \mathrm{~A}$ ) and inlet air flow rate (ranging from $500 \mathrm{sccm}$ to $2800 \mathrm{sccm}$ ), which leads to oxygen excess ratio values ranging from 5 to 11 .

The adjustable parameters of the ANN model (so-called weights) are specified by the vector $\theta$ and are determined from the training data set $Z^{N}$ :

$$
Z^{N}=\{[u(t), y(t)] \mid t=1, \cdots, N\}
$$

where $u(t)$ and $y(t)$ are the set of inputs and the corresponding desired outputs, respectively.

The training phase aims to determine a mapping from the set of training data to the set of possible weights, so that the ANN model produces predictions $\hat{y}(t)$ :

$$
Z^{N} \rightarrow \hat{\theta}
$$

The prediction error approach, which is the strategy applied here, is based on the introduction of a measure of closeness in terms of a mean square error criterion:

$$
V_{N}\left(\theta, Z^{N}\right)=\frac{1}{2 N} \sum_{t=1}^{N}[y(t)-\hat{y}(t \mid \theta)]^{\mathrm{T}}[y(t)-\hat{y}(t \mid \theta)]
$$


The weights are then found as:

$$
\hat{\theta}=\arg \min _{\theta} V_{N}\left(\theta, Z^{N}\right)
$$

The training phase is performed using Levenberg-Marquart algorithm with an error goal of 0.001 . The validation results are presented in Figure 2.

Two criteria, namely root mean square error $\left(\mathrm{RMSE}=\sqrt{\sum_{i=1}^{n}\left(y_{i}-\hat{y}_{i}\right)^{2}} / n\right)$ and absolute maximal error $\left(\mathrm{AME}=\max _{i}\left(\left|y_{i}-\hat{y}_{i}\right|\right)\right)$, are considered to assess the one step ahead prediction performance of the ANN model. $n$ represents the number of data, $y$ and $\hat{y}$ denote experimental and simulated data, respectively.

With an RMSE of 0.003 and an AME under 0.1 the predictive performance accuracy of the ANN model is more than sufficient, especially since it is dedicated to control purposes (Table 1).

\subsection{Parameterized Nonlinear MPC for PEMFC}

In this study, a parameterized NMPC controller is used to control the PEMFC oxygen excess ratio $\left(\lambda_{\mathrm{O}_{2}}\right)$, once the manipulated variable is the air inlet flow rate $\left(\dot{m}_{\text {air }}\right.$ ) and the load current $(I)$ is a measured disturbance.

NMPC is an optimization-based multivariable constrained control method that uses a nonlinear model to predict the future behavior of the process. Classical

Table 1. Training and validation results of the ANN model.

\begin{tabular}{ccc}
\hline & Training & Validation \\
\hline RMSE & $4.726 \times 10^{-4}$ & 0.003 \\
AME & 0.079 & 0.98 \\
\hline
\end{tabular}

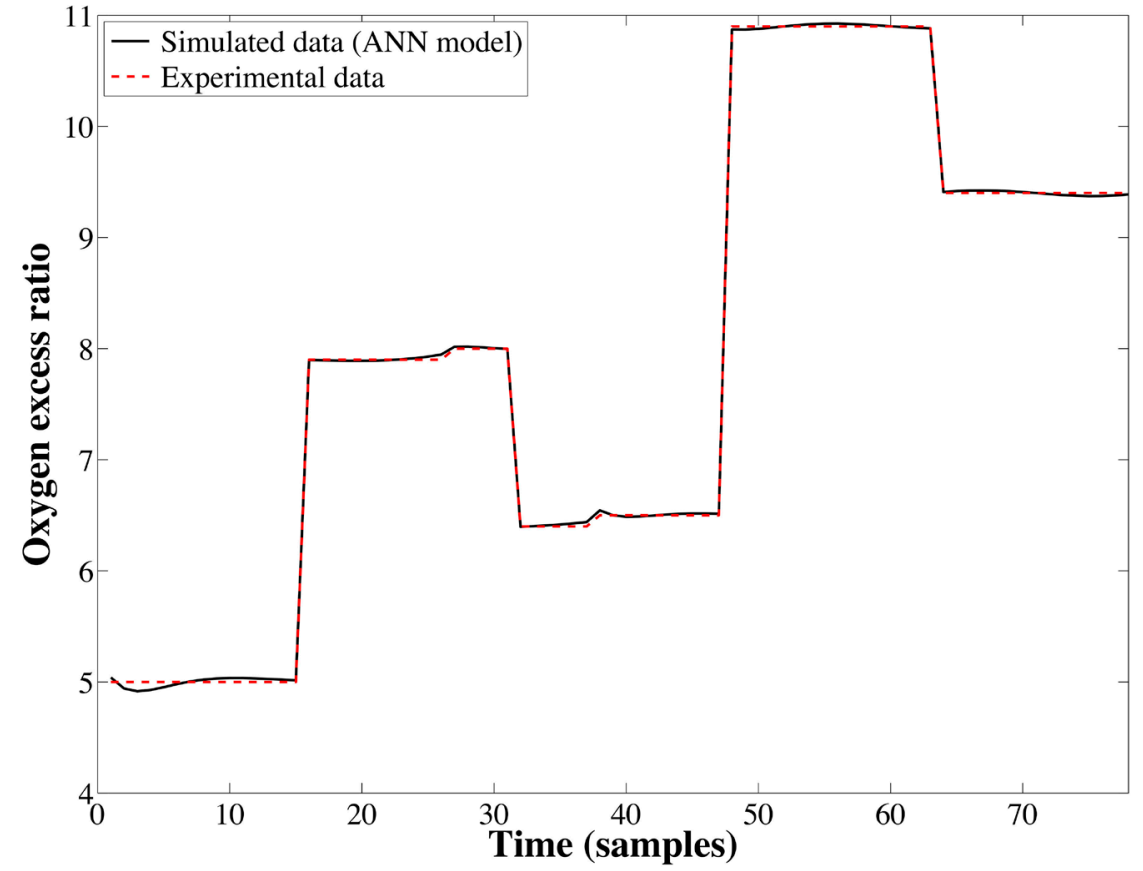

Figure 2. PEMFC ANN model validation. 
NMPC is defined by solving on-line an optimization problem that leads to an optimal sequence of future control actions that minimizes a given cost function over a certain prediction horizon. NMPC offers a great flexibility since it can handle input/state constraints, process nonlinearities, and even economical or operating constraints [36]. However, in their basic form, NMPC strategies involve heavy computations, which could become a bolt to reach on-line implementation.

In this work, to address this problem and reach real-time control goal, a parameterized NMPC strategy is designed. When dealing with classical NMPC strategies, the predictive horizon sets the optimization problem dimension, which could lead to huge problem dimension. The main feature of parameterized NMPC strategy consists in decreasing the optimization problem dimension using a particular parameterization of the control sequence $u$ :

$$
u:=\left(u(p)^{(k+1)}, \cdots, u(p)^{\left(k+N_{y}\right)}\right)
$$

where $u(p)^{(k+1)}$ is the control action at time $k+1, N_{y}$ the predictive horizon and $p$ a set of parameters.

$$
\hat{p}=\arg \min _{p}(J(u(p)))
$$

where the optimal solution $\hat{p}$ is the set of parameters that minimizes the cost function $J$ while meeting the problem constraints.

In this context, as the problem dimension depends on the dimension of $p$, itis straightforwardly linked to the parameterization choice and decoupled from the predictive horizon $N_{r}$. In this manner, a well-structured parameterization scheme may significantly decrease the optimization problem dimension, which is a quite important feature to fulfilreal-time requirements [37]. However, it is important to highlight that there is no universal parameterization that can be applied to any problem. Each problem must be represented using a specific parameterization. More details about parameterized NMPC strategies can be consulted in [38] [39].

Here, since the system is open-loop stable, an approximation of the steady control action is used in the parameterization of the control profile to decrease the degree of freedom of the control profile.

In the first step, several experiments with the fuel cell in open loop are carried out. Even if $\lambda_{\mathrm{O}_{2}}$ depends on several variables, Gruber et al. [15] showed that the stationary behavior of the system mainly relies onair inlet flow rate $\left(\dot{m}_{\text {air }}\right)$ and load current $(I)$. Therefore, during these experiments the stationary behavior is tested with different values for the air inlet flow rate $\left(\dot{m}_{\text {air }}\right)$ and the load current $(I)$.

In a second step, these data are gathered to generate a stationary map correlating the load current, the inlet air flow rate and the oxygen excess ratio.

In a last step, based on this stationary map a simplified model is identified to approximate the steady state control action as a function of the oxygen excess ration and the load current:

$$
u^{*}=a_{1} \lambda_{O_{2}} I
$$


$a_{1}$ is a constant parameter that has to be determined off-line.

In this work, a specific parameterization of the control actions based on the approximation of the steady state control action $u^{*}$ and a single scalar parameter $p$ is proposed:

$$
u(p)^{(k+i)}=u^{*(k+i)}+p E^{*(k+i)} \text { for } i \in\left\{1, \cdots, N_{y}\right\}
$$

$p \in[-1,1]$ is a scalar parameter, $u^{*(k+i)}=a_{1} y_{s p}^{(k+i)} I^{(k+i)}$ is the approximation of the steady control action obtained in stationary steady regime for $\lambda_{\mathrm{O}_{2}}^{(k+i)}$ and $I^{(k+i)}$, and $E^{*(k+i)}$ is the trajectory tracking error obtained using $u^{*(k+i)}$. Specifically, $E^{*(k+i)}=y_{s p}^{(k+i)}-\hat{y}_{A N N}^{*(k+i)}$ and $\hat{y}_{A N N}^{*(k+i)}$ is the ANN model output using $u^{*(k+i)}$ and $I^{(k+i)}$ as inputs.

In this manner, the control action $u(p)^{(k+i)}$ can be seen as a dynamic correction of the approximation of the control action in steady state. In the present case, this is a simple but efficient way to significantly reduce the degree of freedom of the control action profile and so to reduce the computation effort required to solve the optimization problem.

Here the cost function is written to minimize the setpoint tracking error and to limit the variations of the control variable:

$$
J(u(p))=\sum_{i=1}^{N_{y}} \gamma\left(y_{s p}^{(k+i)}-\left(\hat{y}_{A N N}^{(k+i)}+e_{m}^{(k)}\right)\right)^{2}+\sum_{i=1}^{N_{u}} \beta\left(u(p)^{(k+i)}-u(p)^{(k+i-1)}\right)^{2}
$$

$y_{s p}^{(k+i)}$ and $\hat{y}_{A N N}^{(k+i)}$ denote the desired value of oxygen excess ratio and the oxygen excess ratio estimated by ANN model, respectively. $u(p)^{(k+i)}=\dot{m}_{\text {air }}(p)^{(k+i)}$ is the control action at time $k+i$, and $\gamma$ and $\beta$ are two weighting parameters. $N_{y}$ and $N_{u}$ are the control horizon and the predictive horizon, respectively. The prediction horizon corresponds to the future time interval used to compute the process output predictions with the process model. The control horizon corresponds to the time interval when present and future control actions are computed. The control horizon is shorter or equal to the prediction horizon. For time intervals between $N_{u}$ and $N_{y}$ the control actions are constant and set equal to $u(p)^{\left(k+N_{u}\right)}$.

Note that to improve the robustness of the approach against plaint/model mismatch, the error modeling $e_{m}^{(k)}=\hat{y}_{A N N}^{(k)}-y^{(k)}$ at time $k$ is used over the predictive horizon to correct the ANN model outputs $\hat{y}_{A N N}^{(k+i)} \cdot y^{(k)}$ represents the oxygen excess ratio measured at time $k$. Indeed, the cell performance changes along its life, which necessary involves plant/model mismatch. Therefore, to assure an optimal control of the PEMFC along its life, the controller robustness against plant/model mismatch is crucial.

In this context, the optimal value $\hat{p}$ is obtained by minimizing the cost function $J$, which is subject to constraints on manipulated variable:

$$
\hat{p}=\arg \min _{p}(J(u(p)))
$$

Subject to

$$
u(p)^{(k+i)} \in\left[u_{\min }, u_{\max }\right] \text { for } i=1, \cdots, N_{y}
$$


Here, the optimization problem that lead to the sequence of future control actions relies entirely on a single scalar parameter, which dramatically reduces the computational effort compared to a classical NMPC strategy [40].

\section{Experimental Evaluation of the Parameterized Nonlinear MPC}

To assess the performance of the proposed control strategy in terms of tracking capability, disturbances rejection, and robustness against plant-model mismatch, series of experiments are performed on the PEMFC system presented in Section 2. In this aim, several VI's of the SCDA system are modified to replace the built-in controller by the parameterized NMPC controller. With this setup, the optimal control action, computed on-line in $\mathrm{Matlab}^{\star}$ environment, is sent through a specific VI to be applied to the experimental unit.

Here, to exemplify the controller performance three control scenarios are considered: The first scenario illustrates the tracking capability of the controller for a constant value of load current. The second scenario is designed to study the controller ability to cope with disturbances. Eventually, the third scenario evaluates the controller performance in terms of setpoint tracking accuracy in presence of disturbances. A comparison between the built-in controller and the proposed control strategy is made for the second scenario. Note that this comparison cannot be performed for the first and third scenario due to technical constraints. In fact, the built-in controller of the SCDA system, integrated by Fuel Cell Technologies, is designed to operate at constant oxygen excess ratio. In other word, when using the original setup, the oxygen excess ratio value has to be set before the experiments and cannot be modified during the experiments. The built-in controller is a Proportional Integral (PI) controller. However, the SCDA system provided by Fuel Cell Technologies, Inc does not allow to access the controller parameters or to modify them.

In the sequel, the inlet air flow rate is taken as manipulated variable, whereas the load current is a measured disturbance. It is assumed that all other variables, required to ensure safety operation of the fuel cell, are properly controlled. The cell temperature and the sampling period are set to $75^{\circ} \mathrm{C}$ and $3 \mathrm{~s}$ respectively. The optimization problem is solved on-line using Levenberg-Marquardt algorithm. All experiments are performed using control parameters listed in Table 2.

Table 2. Control design parameters.

\begin{tabular}{cc}
\hline Parameter & Value \\
\hline$a_{1}$ & 16.57 \\
$N_{y}$ & 30 \\
$N_{u}$ & 30 \\
$\gamma$ & 1 \\
$\beta$ & 1 \\
\hline
\end{tabular}




\subsection{First Case Scenario: Constant Load Current-Variable Oxygen Excess Ratio Setpoint}

The tracking capability of the controller is evaluated using an oxygen excess ratio setpoint that covers the whole operating condition. In this control scenario, the load current is set to $15 \mathrm{~A}$ and kept constant during the entire experiments. Figure 3 shows that the controller is able to track accurately a variable oxygen excess ratio setpoint, while offering an entirely suitable dynamic for the manipulated variable.

\subsection{Second Case Scenario: Step Changes on Load Current- Constant Oxygen Excess Ratio Setpoint}

To assess the controller performance in terms of disturbances rejection, a set of step changes on the load current is performed. The dynamics of the PEMFC is strongly correlated to the power level. Therefore, to verify that the controller performs accurately whatever the power level, the current steps is chosen to cover the whole operating conditions. Several experiments, with different sets of step changes in load current and different oxygen excess ration values, are carried out to compare the performance of the original built-in controller and the proposed NMPC strategy.

In each and every case, the NMPC controller performed better than the built-in controller. Figure 4 illustrates one of these experiments with the oxygen excess ratio set to 6 .

The NMPC controller demonstrates that it can maintain the oxygen excess ratio to the desired level, even in presence of disturbance. Moreover, compared to the built-in controller, the proposed controller exhibits significantly better disturbances rejection capability. Indeed, for five steps in current $(t=48 \mathrm{~s}, t=138 \mathrm{~s}, t=351 \mathrm{~s}$, $t=438 \mathrm{~s}$ and $t=528 \mathrm{~s})$ an important overshot is observed with the built-in controller, whereas the proposed controller efficiently rejects the disturbances.
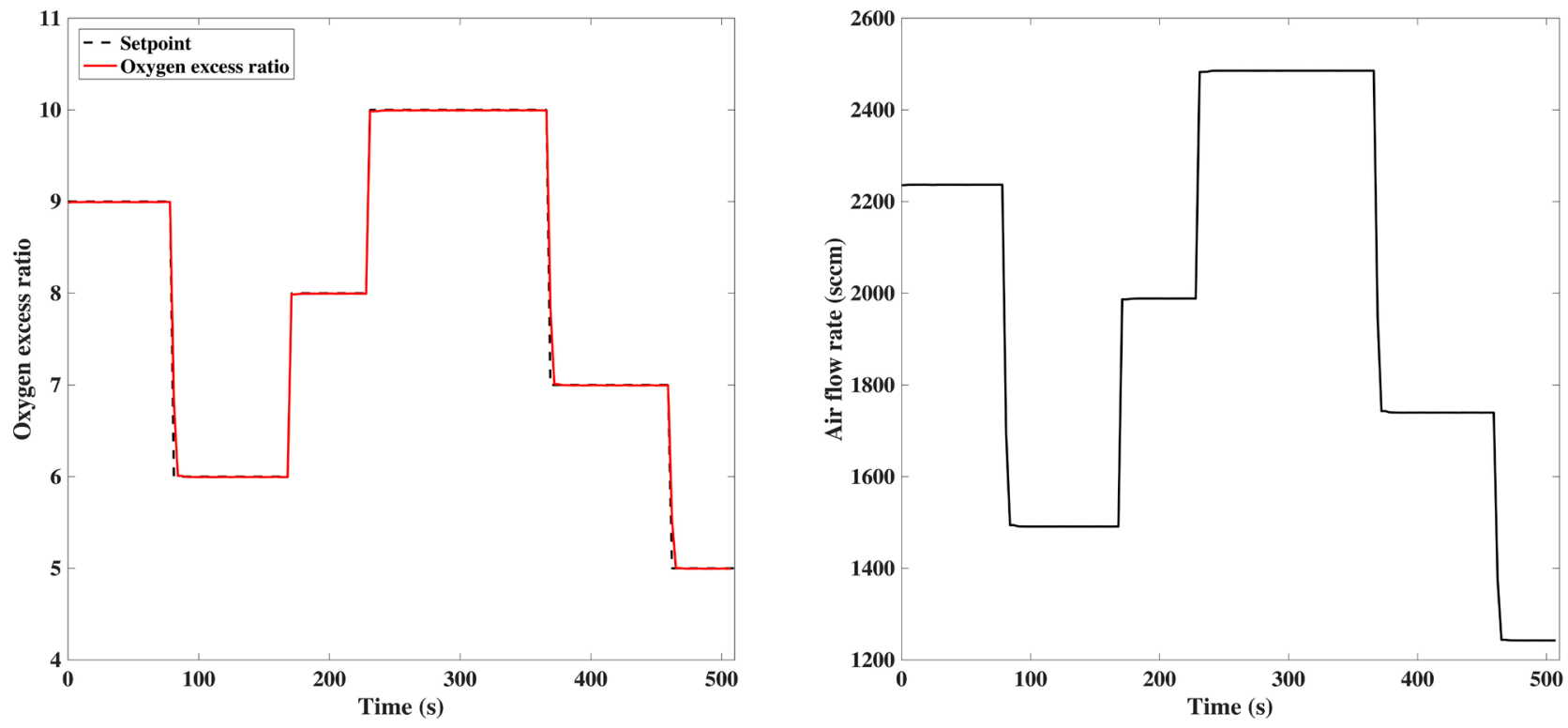

Figure 3. Experimental tracking capability of the proposed controller: (a) oxygen excess ratio; (b) air flow rate. 

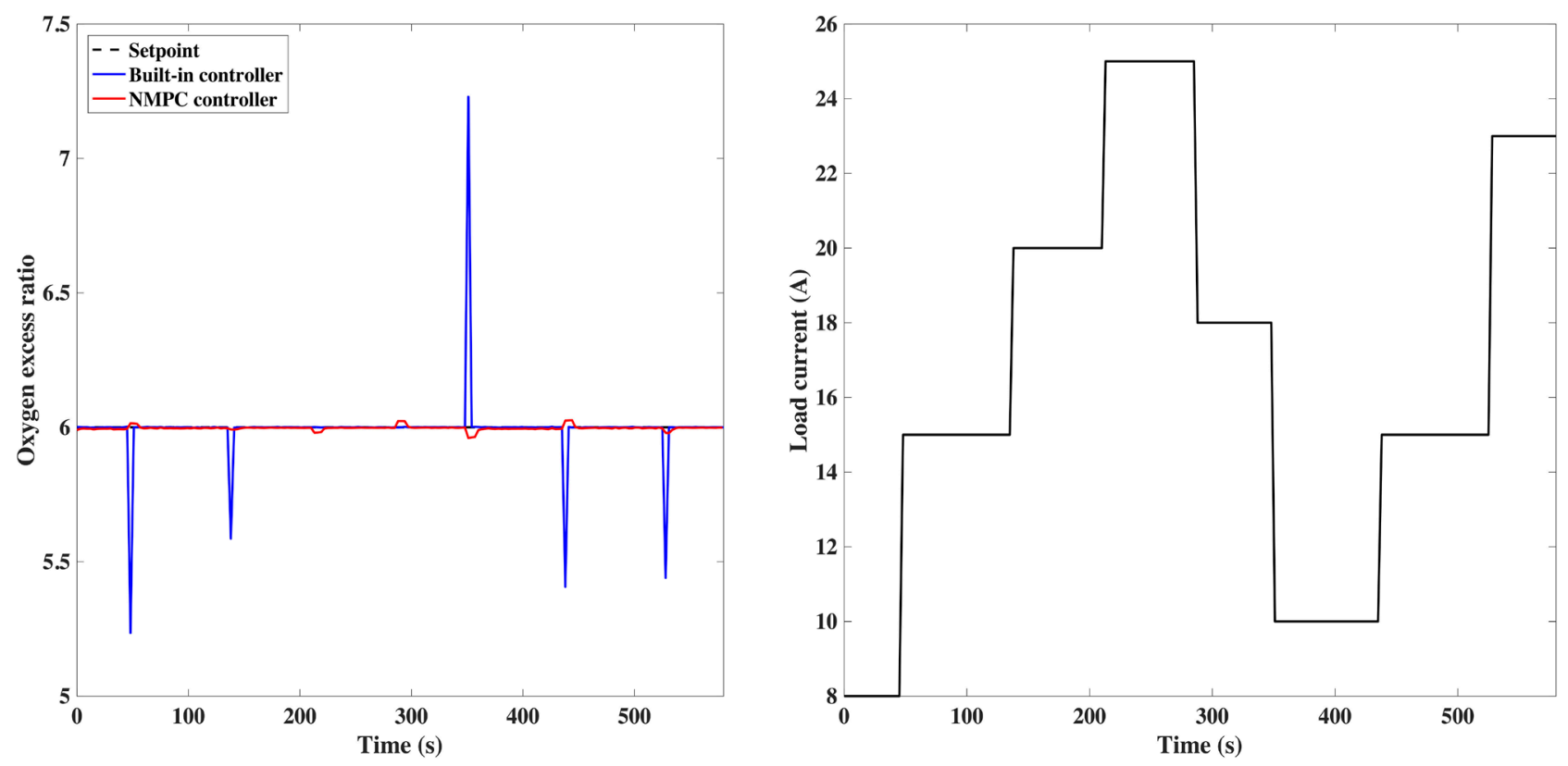

Figure 4. Experimental tracking capability of the proposed controller: (a) oxygen excess ratio; (b) air flow rate.

\subsection{Third Case Scenario: Step Changes on Load Current-Variable Oxygen Excess Ratio Setpoint}

This control scenario is designed to assess the controller performance when the oxygen excess ratio setpoint is modified on-line according to the load current. This control scenario, which is a common working scenario in automotive applications, is significantly important. Indeed, several works demonstrated that updating the oxygen excess ratio reference according to the load current could significantly increase the PEMFC efficiency while avoiding oxygen starvation [16] [41] [42].

The proposed controller demonstrates excellent tracking capability even in presence of disturbances (Figure 5). The ability of the controller to track efficiently an oxygen excess ratio setpoint, calculated according to a variable load current, is a quite important feature. Indeed, this control scenario appears as one of the most promising options to improve the overall PEMFC efficiency, while ensuring safety operation of the fuel cell.

\subsection{Computational Cost}

In order to highlight the benefits of the proposed parameterized NMPC scheme compared to a classical NMPC strategy, both strategies are implemented in Matlab environment and their performance are evaluated in simulation. In this aim, a mechanistic model, which has been presented and validated in a previous work [43], is used as process simulator. To truly highlight the effect of the parametrization of the control sequence, the same ANN model, the same cost function and the same Levenberg-Marquardt algorithm are used for both NMPC controllers. The computational time efficiency is evaluated on the third case scenario 

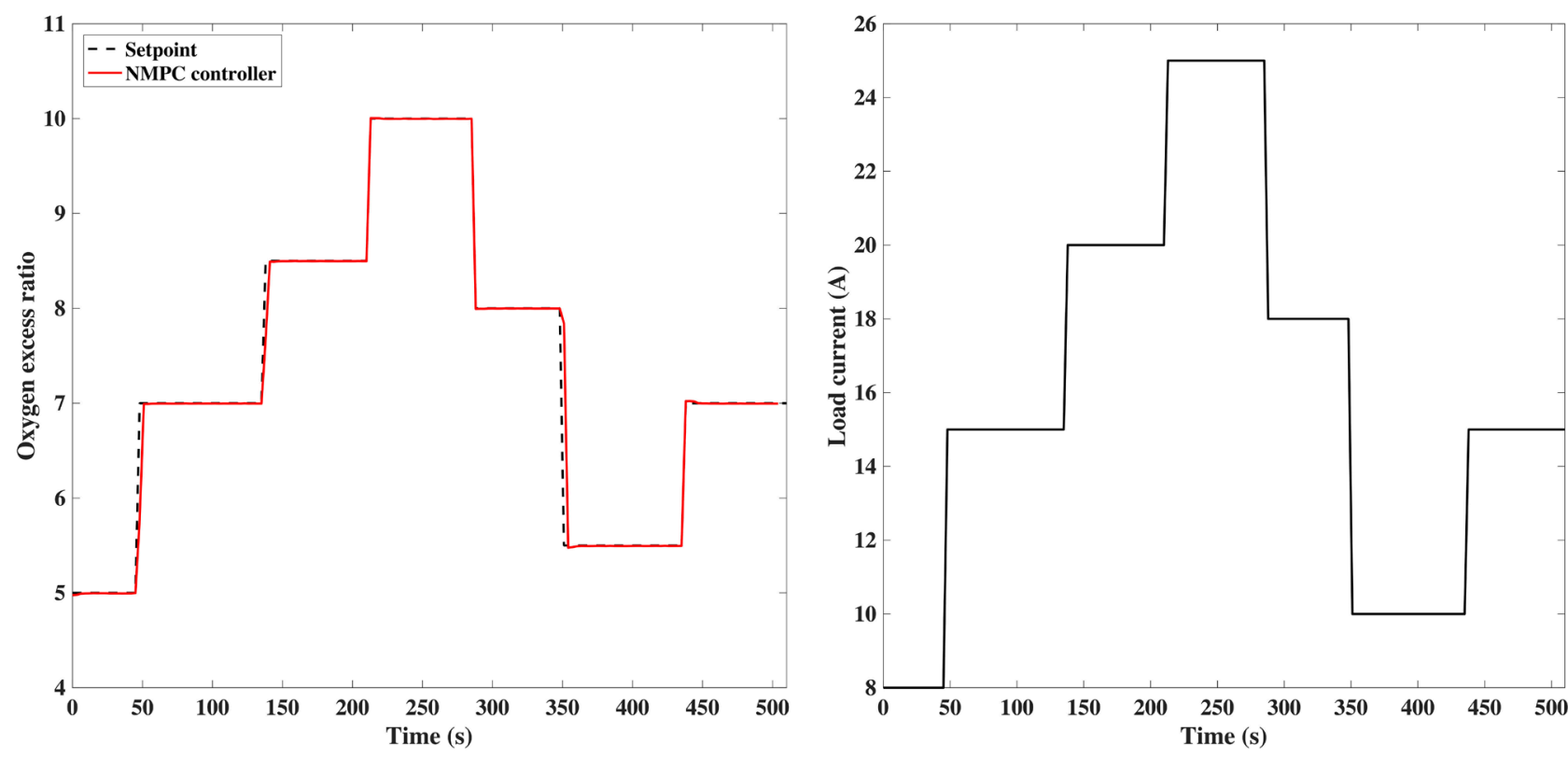

Figure 5. Experimental tracking capability with step changes on load current: (a) oxygen excess ratio; (b) measured disturbances.

(step changes on load current-variable oxygen excess ratio setpoint) for different values of the control horizon $N_{u}$ and the predictive horizon $N_{y}$ : test 1: $N_{y}=N_{u}=10$, test 2: $N_{y}=N_{u}=20$ and test 3: $N_{y}=N_{u}=30$.

The computational effort is evaluated using the mean computational time (MCT) and the maximal computational time $\left(C T_{\infty}\right)$. This last criterion represents the worst case in terms of computational time.

$$
\begin{aligned}
M C T & =\frac{\sum_{i=1}^{n} C T^{(i)}}{n} \\
C T_{\infty} & =\max _{i}\left(C T^{(i)}\right)
\end{aligned}
$$

where $n$ is the number of data and $C T^{(i)}$ the time in second required to solve the control problem at time $t=i$. Note that all simulations are run in Matlab environment on a computer (2.7GHz, RAM@32Go).

Table 3 summarizes the computational time efficiency of both NMPC controllers for all three tests. In all cases, and for similar accuracy performance, the parameterized approach turns out to be significantly faster than the classical NMPC strategy. Whereas the computational time of the classical approach drastically increases with the dimension of the control horizon, the computational time of the parameterized NMPC strategy remains quite the same. The parameterized NMPC approach allows to decouple the optimization problem dimension from the control horizon dimension, which turns out to be quite important feature when dealing with real-time implementation. Considering a control horizon set to 30, which appears to be reasonable regarding a sampling period of $3 \mathrm{~s}$, the parameterized approach is almost 30 times faster than the classical NMPC strategy. Obviously, the computational time depends on what hardware is used to solve the optimization problem. However, the comparison performed here be- 
tween both NMPC approaches clearly demonstrates that the parameterized NMPC controller has a significantly better computation efficiency than the classical NMPC controller.

Note that due to its too high computation cost, the classical NMPC controller has not been experimentally tested on the real fuel cell.

Table 4 summarizes the computational time efficiency of the parameterized NMPC controller for all three cases scenarios presented above in Sections 4.1 to 4.3. These results are obtained experimentally on the real fuel cell.

Regarding the sampling period of 3 seconds, the computational cost of the parameterized NMPC controller appears to be totally acceptable.

It is important to emphasize that in addition to the presented examples, numerous experiments have been carried out in the whole range of operating conditions. In every case, the proposed NMPC controller demonstrated highly satisfactory results in terms of tracking capability and disturbances rejection. Moreover, the computational time of the proposed parameterized NMPC controller is significantly lower than the one of a classical NMCP controller.

Table 3. Comparison between proposed and classical NMPC strategy, in terms of computational time efficiency (simulation results).

\begin{tabular}{|c|c|c|}
\hline & Classical NMPC & Parameterized NMPC \\
\hline test 1: $N_{y}=N_{u}=10$ & 0.804 & 0.102 \\
\hline$M C T(\mathrm{~s})$ & 1.394 & 0.122 \\
\hline \multicolumn{3}{|l|}{$C T_{\infty}(\mathrm{s})$} \\
\hline \multicolumn{3}{|l|}{ test 2: $N_{y}=N_{u}=20$} \\
\hline$M C T(\mathrm{~s})$ & 3.723 & 0.201 \\
\hline$C T_{\infty}(\mathrm{s})$ & 4.918 & 0.215 \\
\hline \multicolumn{3}{|l|}{ test 3: $N_{y}=N_{u}=30$} \\
\hline$M C T(\mathrm{~s})$ & 9.158 & 0.301 \\
\hline$C T_{\infty}(\mathrm{s})$ & 11.446 & 0.322 \\
\hline
\end{tabular}

Table 4. Computational time efficiency (experimental results).

\begin{tabular}{ccc}
\hline & Classical NMPC & Parameterized NMPC \\
\hline test 1: $N_{y}=N_{u}=10$ & 0.804 & 0.102 \\
$M C T(\mathrm{~s})$ & 1.394 & 0.122 \\
$C T_{\infty}(\mathrm{s})$ & & \\
test 2: $N_{y}=N_{u}=20$ & & 0.201 \\
$M C T(\mathrm{~s})$ & 3.723 & 0.215 \\
$C T_{\infty}(\mathrm{s})$ & 4.918 & \\
test 3: $N_{y}=N_{u}=30$ & & 0.301 \\
$M C T(\mathrm{~s})$ & 9.158 & 0.322 \\
$C T_{\infty}(\mathrm{s})$ & 11.446 &
\end{tabular}




\section{Conclusions}

The oxygen excess ratio is considered as a performance variable of the system and its regulation is an important issue since this parameter determines the safety of the fuel cell. In this paper, to address oxygen excess ratio control challenge, a parameterized NMPC strategy has been developed. In a first stage, due to its short computational time and its low sensitivity to noise an artificial neural network (ANN) model has been designed. The oxygen excess ratio is a function of inlet air flow rate, load current, relative humidity of air at the cathode inlet, stack temperature and inlet pressure at the cathode. However, regarding real-time control goal a simplified model has been proposed. This ANN model, used as predictor in the control strategy, is expected to predict the oxygen excess ratio of the PEMFC several steps ahead, once the load current and the inlet air flow rate are available. The validation procedure has been performed on experimental data and the model has shown good performance in terms of prediction accuracy. In a second stage, a parameterized NMPC approach has been designed. This approach, based on a particular parameterization of the control sequence, has led to a low-dimensional optimization problem. Indeed, the optimization problem that leads to the sequence of future control actions relies entirely on a single scalar parameter, which dramatically reduces the computational effort compared to a classical NMPC strategy.

Eventually, the controller has been implemented on-line and experimentally validated on a real fuel cell. Numerous control scenarios have been experimentally conducted to evaluate the controller performance, especially in terms of setpoint tracking accuracy, disturbances rejection and computational cost. These control scenarios gather all the possible scenarios in which the system would have to work. In each and every case, the controller demonstrated highly satisfactory results since it tracked efficiently the desired oxygen excess ratio value while compensating disturbances, regardless of the operating conditions. Besides, experimental comparison demonstrated that the proposed controller had much better disturbances rejection capability than the built-in controller. Eventually, to emphasize the benefit of the proposed controller in terms of computational time efficiency, a comparison has been performed between the proposed NMPC controller and a classical NMPC controller. In all case scenarios, the computational cost of the proposed parametrized NMPC controller was significantly lower than the one of the classical NMPC controller.

Overall, the proposed parameterized NMPC controller appears as an excellent candidate to address the oxygen excess ratio regulation issue.

\section{Conflicts of Interest}

The authors declare no conflicts of interest regarding the publication of this paper.

\section{References}

[1] Larminie, J. and Dicks, A. (2003) Fuel Cell Systems Explained. John Wiley \& Sons 
Ltd., New York. https://doi.org/10.1002/9781118878330

[2] Wang, C. and Nehrir, M.H. (2007) Load Transient Mitigation for Stand-Alone Fuel Cell Power Generation Systems. IEEE Transactions on Energy Conversion, 22, 864-872. https://doi.org/10.1109/TEC.2006.881081

[3] Wang, C., Nehrir, M.H. and Gao, H. (2006) Control of PEM Fuel Cell Distributed Generation Systems. IEEE Transactions on Energy Conversion, 21, 586-595. https://doi.org/10.1109/TEC.2005.860404

[4] Huang, S.R., Lin, C.Y., Wu, C.C. and Yang, S.J. (2008) The Application of Fuzzy Controller for Fuel Cell Generating Studies. International Journal of Hydrogen Energy, 33, 5205. https://doi.org/10.1016/j.ijhydene.2008.05.018

[5] Wang, F.C. and Ko, C.C. (2010) Multivariable Robust PID Control for a PEMFC System. International Journal of Hydrogen Energy, 35, 10437. https://doi.org/10.1016/j.ijhydene.2010.07.111

[6] Methekar, R.N., Prasad, V. and Gudi, R.D. (2007) Dynamic Analysis and Linear Control Strategies for Proton Exchange Membrane Fuel Cell Using Distributed Parameter Model. Journal of Power Sources, 165, 152-170. https://doi.org/10.1016/j.jpowsour.2006.11.047

[7] Li, Q., Chen, W., Wang, Y., Jia, J. and Han, M. (2009) Nonlinear Robust Control of Proton Exchange Membrane Fuel Cell by State Feedback Exact Linearization. Journal of Power Sources, 194, 338-348. https://doi.org/10.1016/j.jpowsour.2009.04.077

[8] Da Fonseca, R., Bideaux, E., Gerard, M., Jeanneret, B., Desbois-Renaudin, M. and Sari, A. (2014) Control of PEMFC System Air Group Using Differential Flatness Approach: Validation by a Dynamic Fuel Cell System Model. Applied Energy, 113, 219-229. https://doi.org/10.1016/j.apenergy.2013.07.043

[9] Hatti, M. and Tioursi, M. (2009) Dynamic Neural Network Controller Model of PEM Fuel Cell System. International Journal of Hydrogen Energy, 34, 5015-5021. https://doi.org/10.1016/j.ijhydene.2008.12.094

[10] Hasikos, J., Sarimveis, H., Zervas, P.L. and Markatos, N.C. (2009) Operational Optimization and Real-Time Control of Fuel-Cell Systems. Journal of Power Sources, 193, 258-268. https://doi.org/10.1016/j.jpowsour.2009.01.048

[11] Methekar, R.N., Patwardhan, S.C., Rengaswamy, R., Gudi, R.D. and Prasad, V. (2010) Control of Proton Exchange Membrane Fuel Cells Using Data Driven State Space Models. Chemical Engineering Research and Design, 88, 861-874. https://doi.org/10.1016/j.cherd.2009.12.001

[12] Golbert, J. and Lewin, D.R. (2004) Model-Based Control of Fuel Cells: Regulatory Control. Journal of Power Sources, 135, 135-151. https://doi.org/10.1016/j.jpowsour.2004.04.008

[13] Wu, W., Xu, J.P. and Hwang, J.J. (2009) Multi-Loop Nonlinear Predictive Control Scheme for a Simplistic Hybrid Energy System. International Journal of Hydrogen Energy, 34, 3953-3964. https://doi.org/10.1016/j.ijhydene.2009.02.060

[14] Shokuhi-Rad, A., Jamali, A., Naghashzadegan, M., Nariman-zadeh, N. and Hajiloo, A. (2012) Optimum Pareto Design of Non-Linear Predictive Control with Multi-Design Variables for PEM Fuel Cell. International Journal of Hydrogen Energy, 37, 11244-11254. https://doi.org/10.1016/j.ijhydene.2012.03.092

[15] Gruber, J., Doll, M. and Bordons, C. (2009) Design and Experimental Validation of a Constrained MPC for the Air Feed of a Fuel Cell. Control Engineering Practice, 17, 874-885. https://doi.org/10.1016/j.conengprac.2009.02.006 
[16] Ziogou, C., Papadopoulou, S., Georgiadis, M.C. and Voutetakis, S. (2013) On-Line Nonlinear Model Predictive Control of a PEM Fuel Cell System. Journal of Process Control, 23, 483-492. https://doi.org/10.1016/j.jprocont.2013.01.011

[17] Nguyen, T.V. and White, R.E. (1993) A Water and Heat Management Model for Proton Exchange Membrane Fuel Cells. Journal of the Electrochemical Society, 140, 2178-2186. https://doi.org/10.1149/1.2220792

[18] Yi, J.S. and Nguyen, T.V. (1998) An along-the-Channel Model for Proton Exchange Membrane Fuel Cells. Journal of the Electrochemical Society, 145, 1149-1159. https://doi.org/10.1149/1.1838431

[19] Um, S., Wang, C.Y. and Chen, K.S. (2000) Computational Fluid Dynamics Modeling of Proton Exchange Membrane Fuel Cells. Journal of the Electrochemical Society, 147, 4485-4493. https://doi.org/10.1149/1.1394090

[20] Wang, Z.H., Wang, C.Y. and Chen, K.S. (2001) Two-Phase Flow and Transport in the Air Cathode of Proton Exchange Membrane Fuel Cells. Journal of Power Sources, 94, 40-50. https://doi.org/10.1016/S0378-7753(00)00662-5

[21] Dutta, S., Shimpalee, S. and Van Zee, J.W. (2000) Three-Dimensional Numerical Simulation of Straight Channel PEM Fuel Cells. Journal of Applied Electrochemistry, 30, 135-146. https://doi.org/10.1023/A:1003964201327

[22] Berning, T., Lu, D. and Djilali, N. (2002) Three-Dimensional Computational Analysis of Transport Phenomena in a PEM Fuel Cell. Journal of Power Sources, 106, 284-294. https://doi.org/10.1016/S0378-7753(01)01057-6

[23] Um, S. and Wang, C.Y. (2004) Three-Dimensional Analysis of Transport and Electrochemical Reactions in Polymer Electrolyte Fuel Cells. Journal of Power Sources, 125, 40-51. https://doi.org/10.1016/j.jpowsour.2003.07.007

[24] Bernardi, D.M. and Verbrugge, M.W. (1992) A Mathematical Model of the Solid-Polymer-Electrolyte Fuel Cell. Journal of the Electrochemical Society, 139, 2477-2491. https://doi.org/10.1149/1.2221251

[25] Springer, T.E., Zawodzinski, T.A. and Gottesfeld, S. (1991) Polymer Electrolyte Fuel Cell Model. Journal of the Electrochemical Society, 138, 2334-2342.

https://doi.org/10.1149/1.2085971

[26] Gurau, V., Liu, H. and Kakac, S. (1998) Two-Dimensional Model for Proton Exchange Membrane Fuel Cells. AIChE Journal, 44, 2410-2422.

https://doi.org/10.1002/aic.690441109

[27] Hu, M., Gu, A., Wang, M., Zhu, X. and Yu, L. (2004) Three Dimensional, Two Phase Flow Mathematical Model for PEM Fuel Cell: Part I. Model Development. Energy Conversion and Management, 45, 1861-1882. https://doi.org/10.1016/j.enconman.2003.09.022

[28] Rowe, A. and Li, X. (2001) Mathematical Modeling of Proton Exchange Membrane Fuel Cells. Journal of Power Source, 102, 82-96. https://doi.org/10.1016/S0378-7753(01)00798-4

[29] Saengrung, A., Abtahi, A. and Zilouchian, A. (2007) Neural Network Model for a Commercial PEM Fuel Cell System. Journal of Power Sources, 172, 749-759. https://doi.org/10.1016/j.jpowsour.2007.05.039

[30] Sisworahardjo, N.S., Yalcinoz, T., El-Sharkh, M.Y. and Alam, M.S. (2010) Neural Network Model of $100 \mathrm{~W}$ Portable PEM Fuel Cell and Experimental Verification. International Journal of Hydrogen Energy, 35, 9104-9109. https://doi.org/10.1016/j.ijhydene.2010.05.124

[31] Chavez-Ramirez, A.U., Munoz-Guerrero, R., Duron-Torres, S.M., Ferraro, M., 
Brunaccini, G., Sergi, F., Antonucci, V. and Arriaga, L.G. (2010) High Power Fuel Cell Simulator Based on Artificial Neural Network. International Journal of Hydrogen Energy, 35, 12125-12133. https://doi.org/10.1016/j.ijhydene.2009.09.071

[32] Arce, A., Ramirez, D.R., del Real, A.J. and Bordons, C. (2007) Constrained Explicit Predictive Control Strategies for PEM Fuel Cell Systems. Proceedings of the 46th IEEE Conference on Decision and Control, Louisiana, LA, 6088-6093. https://doi.org/10.1109/CDC.2007.4434556

[33] Pukrushpan, J.T., Stefanopoulou, A.G. and Peng, H. (2004) Control of Fuel Cell Breathing. IEEE Control Systems Magazine, 24, 30-46. https://doi.org/10.1109/MCS.2004.1275430

[34] Thounthong, P. and Sethakul, P. (2007) Analysis of a Fuel Starvation Phenomenon of a PEM Fuel Cell. Power Conversion Conference, Nagoya, Japan, 731-738. https://doi.org/10.1109/PCCON.2007.373048

[35] del Real, A.J., Arce, A. and Bordons, C. (2007) Development and Experimental Validation of a PEM Fuel Cell Dynamic Model. Journal of Power Sources, 173, 310-324. https://doi.org/10.1016/j.jpowsour.2007.04.066

[36] Richalet, J. (1997) Techniques de l'ingénieur. Informatique Industrielle.

[37] Murilo De Almeida Pinto, A. (2009) Contribution à la Commande Prédictive Non Linéaire pour les Systèmes à Dynamiques Rapides. $\mathrm{PhD}$ Thesis, Institut Polytechnique de Grenoble.

[38] Alamir, M. (2006) Lecture Notes in Control and Information Science. Springer, London.

[39] Amari, R., Alamir, M. and Tona, P. (2008) Proceedings of the IFAC World Congress, South Korea.

[40] Damour, C., Benne, M., Kadjo, A., Rosini, S. and Grondin-Perez, B. (2013) Fast NMPC Scheme of a $10 \mathrm{~kW}$ Commercial PEMFC. International Journal of Hydrogen Energy, 38, 7407-74013. https://doi.org/10.1016/j.ijhydene.2013.04.019

[41] Becherif, M. and Hissel, D. (2010) MPPT of a PEMFC Based on Air Supply Control of the Motocompressor Group. International Journal of Hydrogen Energy, 35, 12521-12530. https://doi.org/10.1016/j.ijhydene.2010.06.094

[42] Santarelli, M.G., Torchioa, M.F., Cali, M. and Giaretto, V. (2007) Experimental Analysis of Cathode Flow Stoichiometry on the Electrical Performance of a PEMFC Stack. International Journal of Hydrogen Energy, 32, 710-716. https://doi.org/10.1016/j.ijhydene.2006.08.008

[43] Damour, C., Grondin, D., Benne, M., Grondin-Perez, B., Deseure, J. and Chabriat, J.P. (2012) Innovative Model-Based Control Approach of a Proton Exchange Membrane Fuel Cell System. Journal of Power Sources, 206, 144-152. https://doi.org/10.1016/j.jpowsour.2012.01.096 